\title{
Article \\ Understanding the Role of Different Substrate Geometries for Achieving Optimum Tip-Enhanced Raman Scattering Sensitivity
}

\author{
Lu He, Mahfujur Rahaman *(1), Teresa I. Madeira and Dietrich R.T. Zahn (1) \\ Semiconductor Physics, Chemnitz University of Technology, D-09107 Chemnitz, Germany; \\ lu.he@s2017.tu-chemnitz.de (L.H.); Teresa.madeira@physik.tu-chemnitz.de (T.I.M.); \\ zahn@physik.tu-chemnitz.de (D.R.T.Z.) \\ * Correspondence: mahfujur.rahaman@physik.tu-chemnitz.de
}

check for updates

Citation: He, L.; Rahaman, M.;

Madeira, T.I.; Zahn, D.R.T.

Understanding the Role of Different Substrate Geometries for Achieving Optimum Tip-Enhanced Raman Scattering Sensitivity. Nanomaterials 2021, 11, 376. https://doi.org/ 10.3390/nano11020376

Academic Editors: Anne Talneau and Maria Losurdo

Received: 20 December 2020

Accepted: 28 January 2021

Published: 2 February 2021

Publisher's Note: MDPI stays neutral with regard to jurisdictional claims in published maps and institutional affiliations.

Copyright: (c) 2021 by the authors. Licensee MDPI, Basel, Switzerland. This article is an open access article distributed under the terms and conditions of the Creative Commons Attribution (CC BY) license (https:/ / creativecommons.org/licenses/by/ $4.0 /)$.

\begin{abstract}
Tip-enhanced Raman spectroscopy (TERS) has experienced tremendous progress over the last two decades. Despite detecting single molecules and achieving sub-nanometer spatial resolution, attaining high TERS sensitivity is still a challenging task due to low reproducibility of tip fabrication, especially regarding very sharp tip apices. Here, we present an approach for achieving strong TERS sensitivity via a systematic study of the near-field enhancement properties in the so-called gap-mode TERS configurations using the combination of finite element method (FEM) simulations and TERS experiments. In the simulation study, a gold tip apex is fixed at $80 \mathrm{~nm}$ of diameter, and the substrate consists of $20 \mathrm{~nm}$ high gold nanodiscs with diameter varying from $5 \mathrm{~nm}$ to $120 \mathrm{~nm}$ placed on a flat extended gold substrate. The local electric field distributions are computed in the spectral range from $500 \mathrm{~nm}$ to $800 \mathrm{~nm}$ with the tip placed both at the center and the edge of the gold nanostructure. The model is then compared with the typical gap-mode TERS configuration, in which a tip of varying diameter from $2 \mathrm{~nm}$ to $160 \mathrm{~nm}$ is placed in the proximity of a gold thin film. Our simulations show that the tip-nanodisc combined system provides much improved TERS sensitivity compared to the conventional gap-mode TERS configuration. We find that for the same tip diameter, the spatial resolution achieved in the tip-nanodisc model is much better than that observed in the conventional gap-mode TERS, which requires a very sharp metal tip to achieve the same spatial resolution on an extended metal substrate. Finally, TERS experiments are conducted on gold nanodisc arrays using home-built gold tips to validate our simulation results. Our simulations provide a guide for designing and realization of both high-spatial resolution and strong TERS intensity in future TERS experiments.
\end{abstract}

Keywords: gap-mode TERS; FEM simulations; metallic nanostructures; plasmonic modes; enhancement factor; spatial resolution

\section{Introduction}

Tip-enhanced Raman spectroscopy (TERS) is a powerful technique that combines Raman spectroscopy with scanning probe microscopy (SPM), i.e., atomic force microscopy (AFM) or scanning tunneling microscopy (STM). TERS thus probes the local vibrational properties of analytes with a spatial resolution far beyond the diffraction limit of incident light. When excited with a suitable light source, the sharp metallic tip confines and enhances the optical field in the vicinity of the tip apex, which then produces Raman scattering from a nanoscopic volume of a sample under the tip. Therefore, by scanning the tip across the sample surface one can realize Raman images of the materials to be probed with an excellent spatial resolution. For example, a resolution of $1.7 \mathrm{~nm}$ for carbon nanotubes [1], $3 \mathrm{~nm}$ for a Pd/Au bimetallic model catalyst [2], and $2.3 \mathrm{~nm}$ for $\mathrm{MoS}_{2}$ [3] in ambient conditions using AFM-TERS and sub-nanometer resolution [4-6] for probing benzene rings via STM-TERS in ultra-high vacuum were reported. 
TERS works based on the principle of electromagnetic (EM) field enhancement around the tip apex, since a metal nanostructure would employ the localized surface plasmon resonance (LSPR) and the lightning rod effect [7]. The lightning rod effect is introduced by the geometric anisotropy of the tip and is independent of the excitation wavelength, while the LSPR is created due to the collective oscillation of free charge carriers in the metal nanostructure [8]. There are many factors that influence the LSPR, such as material, size, shape, and morphology [9]. Therefore, an appropriate selection/modification of these parameters can tune the LSPR over a wide spectral range.

Gold and silver are the two most widely used noble metals for TERS tips due to their small dielectric loss [10], stability in air, and tunability of the LSPR in the visible spectrum. Both metals have some advantages and disadvantages, which become the critical deciding factors for TERS applications. While silver shows a more pronounced plasmonic effect, gold provides a better environmental stability. Consequently, the latter makes gold the more popular material for TERS tips. The two main parameters of TERS compared to conventional Raman spectroscopy are the enhanced signal intensity (also known as enhancement factor (EF)) and the spatial resolution (both together we termed as TERS sensitivity in this work). A usual practice of increasing TERS sensitivity multi-fold compared to conventional TERS is to introduce a metal substrate in order to obtain the so-called gap-mode TERS configuration [11,12]. In this configuration, an image dipole is formed in the metal substrate, thus increasing the sensitivity to a great extent. The EF in a gap-mode TERS experiment can reach up to $10^{6}-10^{8}$ [13] and can be three orders of magnitude higher than that of non-gap-mode TERS [14]. The width of the confined field in the gap-mode TERS configuration is strongly related with the tip radius and the tip-sample gap $[9,15,16]$. Interestingly, as reported by $\mathrm{Xu}$ et al. [9], there is a compromise between the enhancement factor $(\mathrm{EF})$ and the spatial resolution with respect to the tip diameter at resonant excitation. A larger tip radius provides better signal intensity due to the larger scattering cross section at the cost of decreased spatial resolution. Therefore, in order to achieve a better spatial resolution, one needs to decrease either the tip radius or the tip-sample gap. Since decreasing the tip-sample gap below $1 \mathrm{~nm}$ induces non-local quantum effects, which quenches the TERS sensitivity [17], decreasing the tip radius is a more straightforward method to obtain higher TERS sensitivity via combination of LSPR and the lightning rod effect with very little trade-off [18].

However, fabricating sharp tips with good reproducibility to achieve a considerable lightning rod effect is still a big challenge [19-26]. On the other hand, high spatial resolution together with marked EF can be obtained using a metal nanostructured substrate as demonstrated in our previous work [3]. This is advantageous since the fabrication techniques of plasmonic substrates in a reproducible manner with controllable sizes and shapes are well established $[27,28]$. Using such an approach, we can tune the combined tip-substrate plasmonic system with a wide range of parameters and spectral range customized to an existing experimental configuration. Even though some recent works addressed the influence of the substrate on the TERS sensitivity [29,30], a systematic understanding of the effect of substrate size and shape on the TERS sensitivity is still missing. In this work, we at first perform a series of 2D numerical investigations on the evolution of the local enhancement factor and the spatial resolution with respect to the typical gap-mode TERS geometry that consists of a gold tip with varying apex diameter from $2 \mathrm{~nm}$ to $160 \mathrm{~nm}$ on flat gold. Then, a gold nanodisc of $20 \mathrm{~nm}$ height on a flat gold substrate with the nanodisc diameter varying from $5 \mathrm{~nm}$ up to $120 \mathrm{~nm}$ serves as a model for a nanostructured substrate. In this case, the gold tip diameter is fixed at $80 \mathrm{~nm}$. Finally, TERS experiments are performed and the results are compared with the corresponding $3 \mathrm{D}$ simulation.

\section{Materials and Methods}

\subsection{D Numerical Methods and Geometries}

All tip-substrate systems considered in this study are simulated with a tip-substrate gap of $1 \mathrm{~nm}$, below which quantum effects may dominate [31]. Finite element method 
(FEM) simulations are performed in a 2D model to avoid the huge computational cost by 3D simulations in the frequency domain of Wave Optics Module using the COMSOL

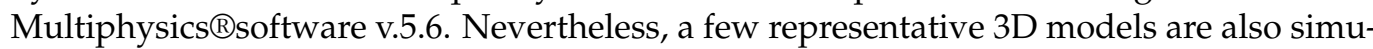
lated for comparison with specific $2 \mathrm{D}$ simulation and experimental results. The electric field is calculated by solving Maxwell's $\nabla \times \mu_{r}^{-1}(\nabla \times \vec{E})-k_{0}^{2}\left(\varepsilon_{r}-\frac{j \sigma}{\omega \varepsilon_{0}}\right) \vec{E}=0$, with $\vec{E}(x, y, z)=\widetilde{E}(x, y) e^{-i k_{z} z}$. The incident optical field is set as a plane wave propagating along the $x$-axis, with $\vec{E}$ varying along the $y$-axis as indicated in Figure 1 .

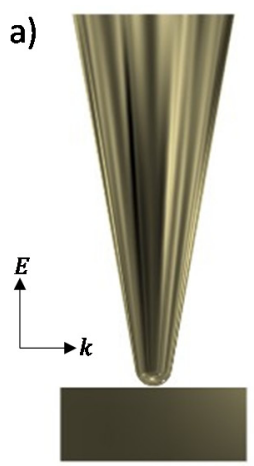

b)

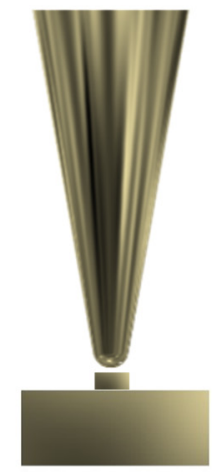

c)

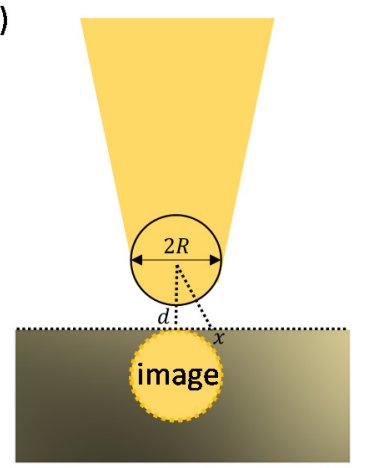

Figure 1. Simulated tip-enhanced Raman spectroscopy (TERS) models consists of (a) conical gold tip with various diameters from $2 \mathrm{~nm}$ to $160 \mathrm{~nm}$ placed on a flat gold film substrate and (b) conical gold tip with fixed diameter of $80 \mathrm{~nm}$ and a flat gold substrate modified by a $20 \mathrm{~nm}$ high gold nanodisc with varying diameter from $5 \mathrm{~nm}$ to $120 \mathrm{~nm}$. (c) Schematic representation of the image dipole formation from the model shown in Figure 1a. The wavevector propagates along the $x$-axis and the $\vec{E}$ vector is along the $y$-axis.

The geometry shown in Figure 1a consists of a conical tip with varying tip apex (diameter from $2 \mathrm{~nm}$ to $160 \mathrm{~nm}$ ) and a flat gold thin film substrate. This geometry is representative for conventional gap-mode TERS and is thus simulated to benchmark our simulations as this configuration was already studied in detail [9,32-34]. To investigate the influence of the substrate geometry on TERS sensitivity, a gold nanodisc having a 2D-cross section of rectangular shape is added to the flat gold thin film with its diameter varying from $5 \mathrm{~nm}$ to $120 \mathrm{~nm}$ (see Figure 1b). The height of such nanodisc is fixed at $20 \mathrm{~nm}$ to be consistent with the following TERS experiment. Moreover, the edge of such a nanostructure in a real situation should always possess some curvature rather than a single sharp point. Therefore, we set a roundish shape at the corner of the rectangle with a constant curvature of $2 \mathrm{~nm}$. The diameter of the conical metal tip apex is fixed at $80 \mathrm{~nm}$ in this configuration to be consistent with the experiment.

A rectangular perfect matched layer (PML) is used in all simulations to absorb the light scattered from the boundary [35]. The amplitude of the electric field of the excitation light is set at $1 \mathrm{~V} / \mathrm{m}$. An optimized mesh is used after a convergence test in COMSOL, and the minimum element size in the gap area is set at $0.1 \mathrm{~nm}$. A non-uniform mesh is used in the remaining calculated area to balance the performance and the computation time with the maximum element size set as $\lambda / 5 n$, in which $\lambda$ is the excitation wavelength and $n$ is the refractive index. The refractive index of gold used in the simulations is taken from ref. [36].

The spectral dependence is obtained via a wavelength sweep from $500 \mathrm{~nm}$ to $800 \mathrm{~nm}$ for each specific configuration. The maximum TERS EF $\left(E_{l o c}^{4} / E_{0}^{4}\right)$ for each configuration is taken under the resonance condition where the local field has the maximum spectradependent value. The local electric field enhancement factor is calculated at the middle point of the tip-substrate gap.

An analysis of the spatial resolution could be performed using the analogue sketch shown in Figure 1c, which represents the conventional gap-mode TERS configuration as shown in Figure 1a. The drop in potential between the two spheres (original and image 
dipoles) can be expressed as $\Delta V=\left|E_{l o c}\right| d$, while the potential difference between these two sites in the absence of two metal spheres can be expressed as $\Delta V=\left|E_{0}\right|(2 R+d)$, where $E_{0}$ is the incident field and $E_{l o c}$ is the local field. Since the two spheres can be considered as equipotential bodies, we can write $\Delta V=\left|E_{0}\right|(2 R+d)=\left|E_{l o c}\right|$ d. In a certain specific geometry where $R$ and $d$ are fixed. Thus, the lateral offset of electric field from the center can be written as

$$
\left|E_{l o c}(x)\right|=\frac{\Delta V}{2 R+d-2 \sqrt{R^{2}-x^{2}}}
$$

The full-width-at-half-maximum (FWHM) of the local field is thus given by $w=$ $2 \sqrt{R d}[15]$.

Using a similar approach considering the TERS intensity as the 4th power of the local field amplitude, we can write the lateral offset of the TERS signal as

$$
\left|E_{l o c}(x)\right|^{4}=\Delta V^{4} /\left(2 R+d-2 \sqrt{\left(R^{2}-x^{2}\right)}\right)^{4}
$$

Therefore, for a very small $d$, we can obtain the FWHM of the TERS intensity distribution as

$$
\mathrm{FWHM}=2 \sqrt{((\sqrt[4]{2}-1) R d)} \approx 0.87 \sqrt{R d}
$$

\subsection{D Numerical Methods and Models}

3D simulations employing the TERS experimental geometry are performed for comparison with the 2D simulation and the experimental results. The geometry consists of a conical gold tip with a diameter of $80 \mathrm{~nm}$ either placed on a flat gold substrate or on the edge of a gold nanodisc with height of $20 \mathrm{~nm}$ and diameter of $100 \mathrm{~nm}$. $638 \mathrm{~nm}$ excitation is used at an incident angle of $65^{\circ}$ in the $x z$-plane. The light is a $p$-polarized plane wave with its polarization direction and the wavevector are set to be parallel and perpendicular to the tip along axis, respectively. The optical constants of gold used are the same as in the 2D model. An optimal mesh is used with the minimum element size of $0.5 \mathrm{~nm}$. A perfect matched layer is used to absorb the light scattered from the boundary.

\subsection{Experimental Materials and Methods}

TERS experiments are performed using home-made gold coated tips. For this purpose, $100 \mathrm{~nm}$ of gold is evaporated onto commercially available silicon tips using thermal evaporation resulting in the formation of gold clusters at the tip apex. Details of the tip preparation can be found in ref. [37]. The typical diameter of these clusters at the apex are between 80 and $120 \mathrm{~nm}$. The substrate consists of gold nanodiscs on a continuous gold film of $50 \mathrm{~nm}$ on a Si substrate. The diameter of the nanodiscs is $100 \mathrm{~nm}$ with a height of $20 \mathrm{~nm}$ and the distance between two nanodiscs from centre to centre is $150 \mathrm{~nm}$. An ultrathin film of $2 \mathrm{~nm}$ cobalt phthalocyanine $(\mathrm{CoPc})$ is deposited on the plasmonic substrate as Raman probe using organic molecular beam deposition.

TERS mapping is performed on a NanoRaman Platform from HORIBA Scientific (formerly AIST-NT) in side-illumination geometry using a wavelength of $638 \mathrm{~nm}$ for excitation. Incident and scattered light are collected by a long working distance objective $\left(100 \times, 0.7\right.$ N.A.) inclined at an angle of $65^{\circ}$ with respect to the normal of the sample surface. During the TERS measurements the AFM tip operates in intermittent contact mode while the TERS signal is acquired when the tip is in contact with the sample and the contact time is defined by the acquisition time of $0.3 \mathrm{~s}$. An electron-multiplying charge-coupled device (CCD) is used to collect the scattered light signal dispersed by a $600 \mathrm{~L} / \mathrm{mm}$ grating. The step size and the laser power used to acquire a TERS map is $10 \mathrm{~nm}$ and $1 \mathrm{~mW}$, respectively. 


\section{Results and Discussion}

\subsection{D Simulation Results}

3.1.1. Simulation Results on Conventional Gap-Mode TERS Configuration Shown in Figure 1a

Starting with the conventional gap-mode TERS configuration (see Figure 1a), a gold tip with varying diameter from 2 to $160 \mathrm{~nm}$ is placed on a flat gold substrate. This gap-mode TERS configuration is a well-studied system using FEM simulations and a handful publications can be found investigating the effect of different parameters on TERS enhancement [9,32-34]. Surprisingly, we did not find any systematic study of the influence of the tip diameter on the spatial resolution.

Figure 2 shows our simulation results for the conventional gap-mode TERS configuration. The overall spectral dependence of the TERS enhancement with respect to the tip diameter is presented in Figure 2a. The corresponding maximum TERS EF, i.e., TERS EF value at the resonance wavelength, becomes high for both very small and very large tip diameters, as can be clearly seen in Figure $2 b$. As the tip diameter increases from $30 \mathrm{~nm}$ to $160 \mathrm{~nm}$, the TERS enhancement increases strongly. This trend of increasing TERS $\mathrm{EF}$ is due to the combination of two counter-effects, namely increasing scattering cross section and radiative damping, especially at higher tip diameters [38-40]. The gradual red shift of the max. TERS EF position also takes place in this range and originates from the size dependent LSPR effect similar to that observed in previous works [41-43]. In the lower tip diameter regime, the TERS EF increases at a much faster rate with decreasing tip diameter from $28 \mathrm{~nm}$ to $2 \mathrm{~nm}$. The sharp rise of TERS EF is due to the lightning rod effect, because of a geometrical singularity-induced spatial confinement of the surface charge density at the apex. Our 2D simulation results are qualitatively in agreement with previous reports simulating similar geometries using a 3D-FDTD method [9]. In their study, the tip radius-dependent maximum electric field enhancement initially experiences a rapid decrease as the tip diameter increases from $10 \mathrm{~nm}$ to $30 \mathrm{~nm}$ (in our simulation from $2 \mathrm{~nm}$ to $30 \mathrm{~nm}$ ) due to the lightning rod effect. Afterwards, the local electric field increases slowly from $30 \mathrm{~nm}$ to $100 \mathrm{~nm}$ (in our simulation from $30 \mathrm{~nm}$ to $160 \mathrm{~nm}$ ). Further increase of the tip diameter does not provide additional enhancement, as it saturates beyond $100 \mathrm{~nm}$ in their study. However, we do not reach saturation in our 2D simulations. This could be due to the different geometry used in our 2D model and the 3D model simulated in the ref [9].
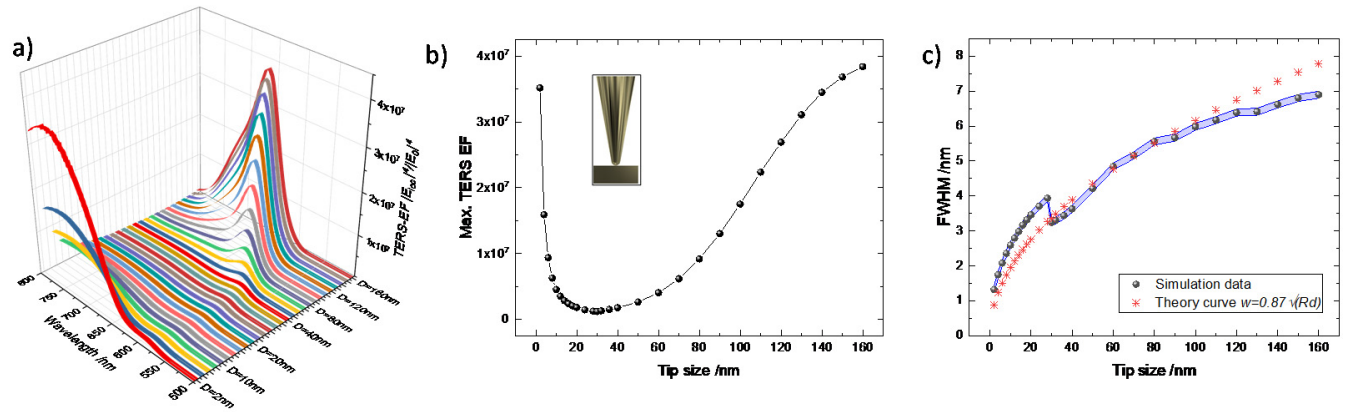

Figure 2. Simulation results of (a) the spectral dependence of the TERS EF, (b) max. TERS EF, and (c) full-width-at-halfmaximum (FWHM) of the local TERS profile in the conventional gap-mode TERS geometry as shown in the inset of Figure $2 \mathrm{~b}$. The simulated FWHM as a function of the tip diameter is compared with the FWHM profile derived from Equation (3) (red asterisks in Figure 2c). The blue shaded area presents the error bar of the FWHM.

Interestingly, the resonance wavelength in the lightning rod effect regime shows a red shift with decreasing tip diameter in agreement with previous work [44-47]. This might be due to the electrical conductivity of the tip material at the excitation energy [48]. It is important to mention here that our observed red shift of the resonance frequency in the regime dominated by the lightning rod effect agrees well with the classical approach [49]. However, several recent quantum mechanical approaches calculated for sub-nanometer 
gap dimers predicted an opposite trend of the resonance frequency in the lightning rod effect regime [17,50-52].

To determine the spatial resolution, the full-width-at-half-maximum (FWHM) of the TERS EF profile in the middle of the gap is derived using a Gauss function. The simulated FWHM is then plotted as a function of the tip diameter in Figure $2 \mathrm{c}$ as indicated by black dots with the blue area representing the error bar obtained from the fitting. We purposefully set the error bar as $0.1 \mathrm{~nm}$ in the cases when the mathematical fit error goes below $0.1 \mathrm{~nm}$, as the physical limit cannot go below this value. Initially, we can see an exponential increase of the FWHM as a function of tip diameter up to a value of $28 \mathrm{~nm}$ and then encounter a sharp drop. Interestingly, this sharp drop occurs at $28 \mathrm{~nm}$ where the maximum TERS EF has its minimum (see Figure 2b). This behavior indicates that the spatial confinement induced by the lightning rod effect is different from the FWHM of the plasmonic field stemming from the dipole-dipole interaction of LSPR mechanism. Comparing Figure $2 b, c$, it is thus evident that below a tip diameter of $28 \mathrm{~nm}$, the lightening rod effect dominates the local field confinement mechanism, while above $28 \mathrm{~nm}$ of tip diameter the lightning rod effect becomes less significant due to the loss of the sharpness and the LSPR controls the plasmonic enhancement. The FWHM derived for a dipole-dipole system using Equation (3) is also plotted in Figure 2c shown by the red asterisks. As can be seen, our simulated FWHMs agree well with the estimated FWHMs beyond the tip diameter of $28 \mathrm{~nm}$ up to 110 $\mathrm{nm}$. Further increasing the tip diameter size shows a smaller FWHM value compared with the estimation using Equation (3). This could be due to the decreasing electromagnetic energy loss of the structure in this range (see supplementary information Figure S1).

\subsubsection{Results on Tip Nanodisc Gap-Mode TERS Configuration Shown in Figure 1b}

In this configuration, the gold tip diameter is kept constant at $80 \mathrm{~nm}$. The diameter of gold nanodisc varies from $5 \mathrm{~nm}$ to $120 \mathrm{~nm}$ with a constant height of $20 \mathrm{~nm}$. The simulated results for the model shown in Figure $1 \mathrm{~b}$ are presented in Figure 3.
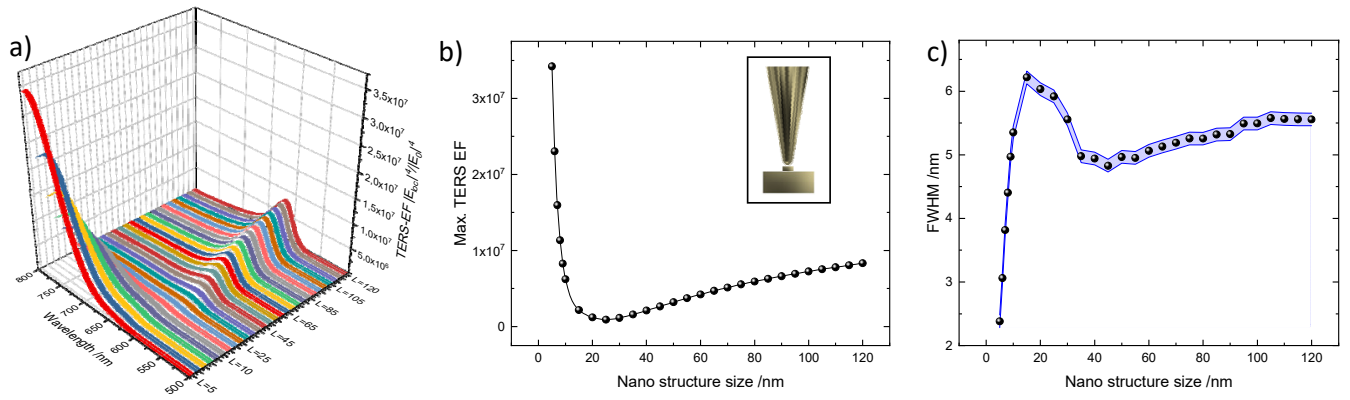

Figure 3. Simulation results when the tip is placed at the center of a nanodisc. (a) Spectral dependence of TERS EF, (b) maximum TERS EF, and (c) FWHM of the TERS EF profile. The blue shaded area presents the error bar of the FWHM. The tip-substrate geometry is shown in the inset of Figure $3 b$.

Figure 3a,b depict the spectral response of the TERS EF and maximum TERS EF as a function of nanodisc diameter with the tip at the center of the nanodisc. Similar to the previous configuration, the maximum TERS EF decreases rapidly as the nanodisc diameter increases from $5 \mathrm{~nm}$ to $25 \mathrm{~nm}$ due to the weakening of the coupling between the tip and the edges of the nanodisc as the edges move further away from the central tip position. However, further increasing the nanodisc diameter above $25 \mathrm{~nm}$ only leads to a moderate increase of the maximum TERS EF as can be seen in Figure $3 \mathrm{~b}$. This is because of the fixed tip diameter $(80 \mathrm{~nm})$, which dominates the resultant scattering cross-section of the image dipole formed at tip-substrate system. Hence, the overall TERS EF in the LSPR regime is weaker for the nanostructured substrate. However, in the lightning rod effect regime, the nanodisc significantly overpowers the gap-mode TERS EF (note that minimum tip diameter and nanodisc diameter in Figures $2 b$ and $3 b$ are $2 \mathrm{~nm}$ and $5 \mathrm{~nm}$, respectively). For example, the combination of a tip of $80 \mathrm{~nm}$ diameter and nanodisc diameter of $5 \mathrm{~nm}$ provides a 
TERS EF of $3.5 \times 10^{7}$, which requires a $2 \mathrm{~nm}$ tip in the gap-mode TERS configuration. More importantly, to achieve this value, one needs to overcome some significant technological challenges related to the fabrication of such extremely sharp tips. On the contrary, thanks to the practical development, well-defined nanostructured substrates can be fabricated using electron beam lithography. Consequently, gold nanodiscs on gold films are favorable for achieving improved TERS EF.

To understand how the nanodisc diameter influences the spatial resolution, we extracted the FWHM of the spatial distribution of maximum TERS EF using the same procedure as above. The resultant FWHM vs. nanodisc diameter is plotted in Figure 3c. As can be seen, the FWHM for the nanostructured substrate configuration behaves differently compared to the gap-mode TERS configuration. Again, one can easily identify two regimes, which are dominated by the lightning rod effect and LSPR, respectively. The FWHM in the lightning rod effect regime is found to be between $2.3 \mathrm{~nm}$ and $6 \mathrm{~nm}$ within the range of the nanodisc diameter from $5 \mathrm{~nm}$ to $20 \mathrm{~nm}$. Comparing Figures $2 \mathrm{c}$ and $3 \mathrm{c}$, we can see that the FWHM in this region in Figure $3 \mathrm{c}$ is larger than that in Figure 2c. This is because when the nanodisc diameter gets considerably smaller (in our simulation below $20 \mathrm{~nm}$ ) than the tip diameter, the coupling between the tip and the two edges becomes stronger and thus enlarges the overall spatial distribution. In the region of nanodisc diameter from $20 \mathrm{~nm}$ to $40 \mathrm{~nm}$, instead of a sharp drop shown in Figure 2c, we observe a gradual switching from the lightning rod effect regime to the LSPR regime since the increasing nanodisc diameter weakens the coupling between the tip and the edges of the nanostructures. Nevertheless, we can see the nanostructured substrate performs better in terms of TERS sensitivity if we compare the two configurations. For example, $6 \mathrm{~nm}$ of tip diameter in the first configuration provides a TERS EF of $1 \times 10^{7}$ and a FWHM of $2 \mathrm{~nm}$, whereas a nanodisc diameter of $5 \mathrm{~nm}$ with a tip diameter of $80 \mathrm{~nm}$ gives a TERS EF of $3.5 \times 10^{7}$ and a FWHM of $2.3 \mathrm{~nm}$.

In our previous work, we demonstrated experimentally that edges of nanostructures can produce even stronger TERS sensitivity [3,53-55]. Therefore, the nanostructured substrate configuration was further tested to understand how the dimension of the nanostructure influences the TERS sensitivity when the tip is located at the edges. The calculated max. TERS EF and the FWHM as a function of the nanodisc diameter are presented in Figure $4 a, b$. The spectral dependence of the TERS EF can be found in the supplementary information (Figure S2). It is important to mention here that we kept the curvature of the edges at $2 \mathrm{~nm}$ for all nanodiscs considered in the simulations.
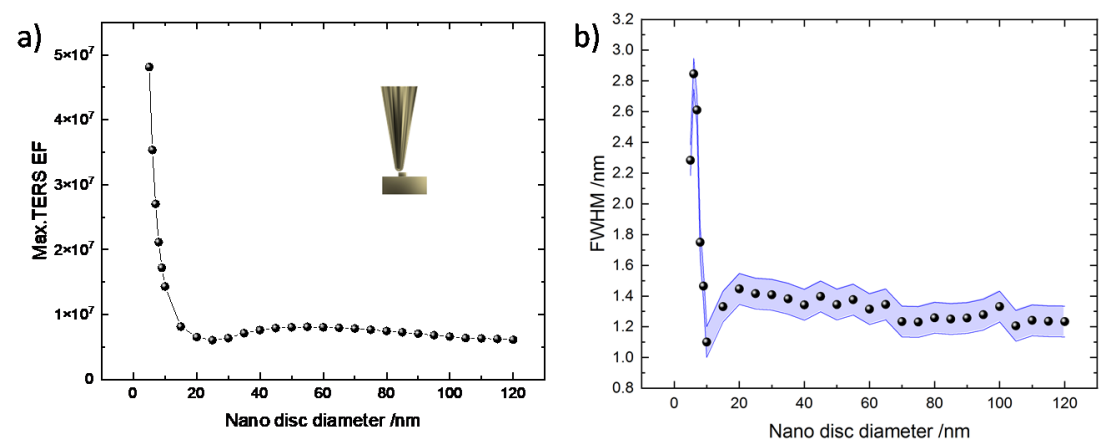

Figure 4. Simulation of TERS EF when the tip is placed at the edge of a nanodisc. (a) Maximum TERS EF and (b) FWHM of the TERS intensity. The geometry is presented in the inset of Figure 4a. The blue shaded area presents the error bar of the FWHM in Figure $4 \mathrm{~b}$.

Comparison between Figures $3 b$ and $4 a$ reveals that the maximum TERS EF is further amplified especially in the range of nanodisc diameters from 5 to $10 \mathrm{~nm}$ where the lightning rod effect is assumed to be the strongest. The impact of the lightning rod effect is observable up to $20 \mathrm{~nm}$ of nanodisc diameter, while further increasing nanodisc diameter does not influence the TERS EF significantly. This is also visible in Figure 4b, where the FWHM remains constant within the fitting uncertainty above $20 \mathrm{~nm}$ of nanodisc diameter. However, 
when decreasing the nanodisc diameter below $10 \mathrm{~nm}$, we observe an increase of the FWHM, most likely due to coupling with the opposite edge of the nanodisc. The smallest FWHM achieved in this configuration is $1.1 \mathrm{~nm}$ for a nanodisc diameter of $10 \mathrm{~nm}$. This is certainly a further improvement compared to the two other configurations discussed above. More importantly, the TERS EF of $1.6 \times 10^{7}$ achieved at this nanodisc diameter for a tip placed at the edge is significantly better than the two configurations discussed above with equivalent dimensions. For $6 \mathrm{~nm}$ of tip diameter and $5 \mathrm{~nm}$ of nanodisc diameter, we obtain a TERS EF and FWHM of $1 \times 10^{7}$ and $2 \mathrm{~nm}$ (tip on flat gold thin film), $3.5 \times 10^{7}$ and $2.3 \mathrm{~nm}$ (tip at the centre of nanodisc), and $5 \times 10^{7}$ and $1.8 \mathrm{~nm}$ (tip at the edge of nanodisc), respectively. Therefore, our 2D simulations clearly demonstrate a path to improve the TERS sensitivity, which is critical and highly sought after in optical nanospectroscopy.

\subsection{Experimental Results and 3D Simulation}

TERS experiments are performed on a nanostructured substrate consisting of a gold nanodisc array on a gold coated silicon substrate to compare with our simulation. An ultrathin $(\sim 2 \mathrm{~nm})$ film of cobalt phthalocyanine (CoPc) is deposited on this plasmonic substrate as a Raman probe. Figure $5 \mathrm{a}, \mathrm{b}$ present the TERS map and AFM topography taken simultaneously using a home-made gold coated TERS tip. The step size of the measurements is $10 \mathrm{~nm}$. Two representative TERS spectra, one on the flat gold surface (green dots in Figure 5a,b) and another one at the edge of the gold nanodisc (blue dots in Figure 5a,b) are shown in Figure $5 c$ with corresponding colors. The TERS map is created for the most intense CoPc Raman peak at $1535 \mathrm{~cm}^{-1}$ as shown by the red shaded area in Figure 5c. As can be seen in Figure 5a, the highest TERS enhancement originates predominantly from the edges of the nanodiscs as predicted by the simulation above. The modified AFM topography in Figure $5 b$ is most likely due to the imperfect profile of the tip apex with multiple clusters contributing to the topography convolution. Nevertheless, from Figure $5 \mathrm{a}$ it is evident that the imperfect profile of the apex has less influence on the TERS map, meaning that coupling between the gold clusters and the nanodisc dictates the TERS mapping. Comparing the TERS spectra shown in Figure $5 c$, it is evident that peak intensities of $\mathrm{CoPc}$ from the nanodisc edge are stronger than from the flat gold. For example, the peak intensity of the phonon mode centered at $1535 \mathrm{~cm}^{-1}$ is about 4.1 times larger than that from the flat gold surface. Our 3D simulations with respect to these two geometries with the same excitation of $638 \mathrm{~nm}$ are shown in Figure 6. It can be seen that the electric field enhancement factor in Figure 6a, representing the geometry of the tip on flat gold, is 46.1 , corresponding to the TERS EF of $4.5 \times 10^{6}$. On the other hand, the electric field EF shown in Figure $6 \mathrm{~b}$ representing the geometry of the tip on the edge of nanodisc, is 65.8 , corresponding to the TERS EF of $1.9 \times 10^{7}$. Thus, the relative EF between the flat gold and the nanodisc edge is calculated to be 4.2. This is in a very good quantitative agreement to the TERS experimental result.

In addition, TERS mapping is also performed on other gold nanostructured substrates consisting of monolayer $\mathrm{MoS}_{2}$ on gold nanodisc arrays on Si (see Figure S3). In this experiment, $\mathrm{MoS}_{2}$ is used as the Raman probe. Here, it is even more evident that the maximum TERS enhancement originates from the edge of the nanodiscs, demonstrating good agreement with the current simulation results. Furthermore, our previous study also confirmed qualitatively the result from the 2D simulation [3,55]. However, we performed a detailed systematic computational study in the present investigation and report the influence of the coupling behaviour between the tip and the plasmonic substrate on TERS sensitivity, which is missing in the previous work [3,55]. 
a)

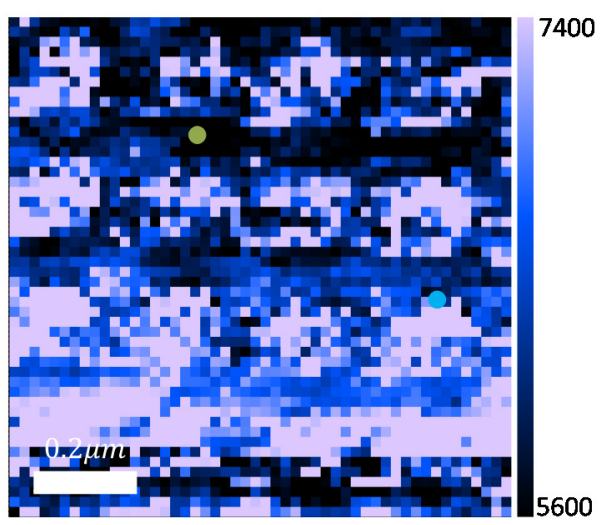

b)

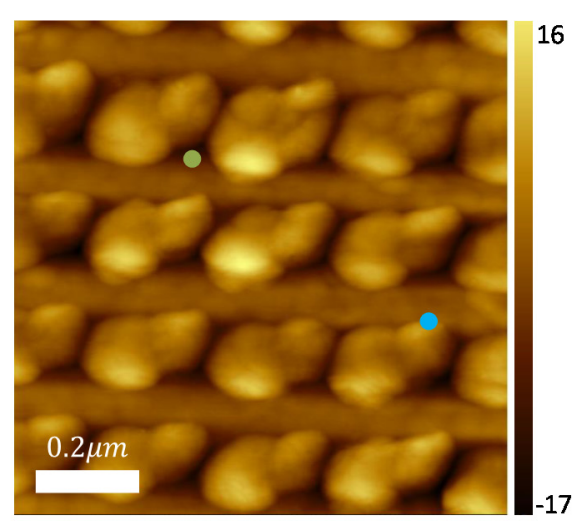

c)

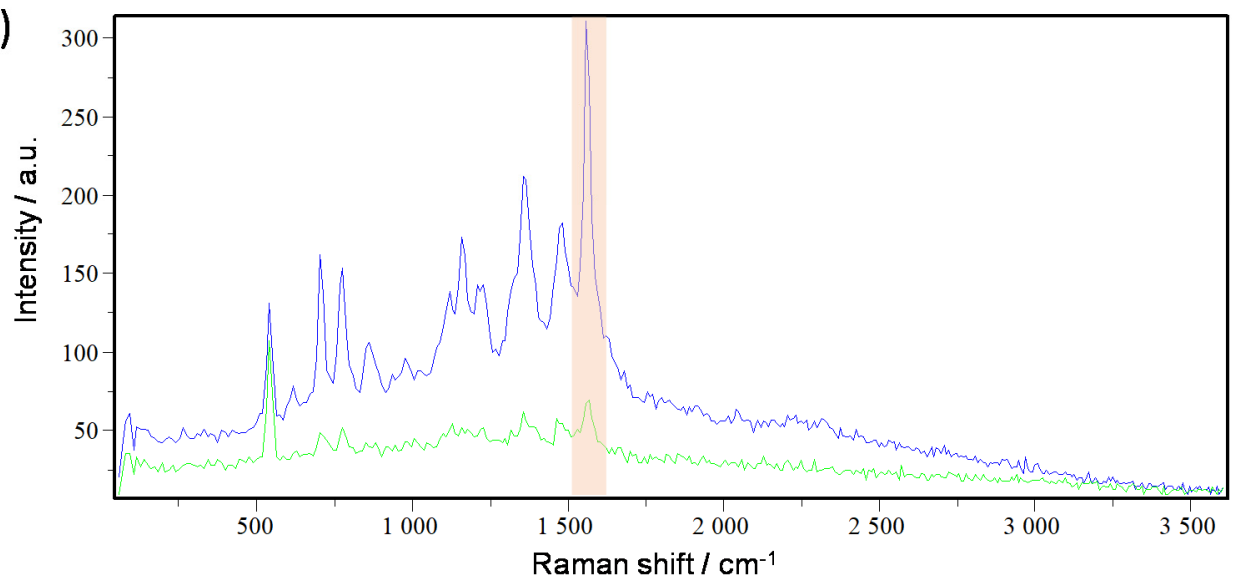

Figure 5. TERS experiment on the reference nanodisc-modified gold substrate. (a) TERS map, (b) Atomic-force microscopy AFM topography, and (c) corresponding Raman spectra taken from two different positions marked by blue and green dots in the TERS map. The TERS map is created using the most intense peak around $1535 \mathrm{~cm}^{-1}$ as shown by the red shaded region in Figure $5 c$.
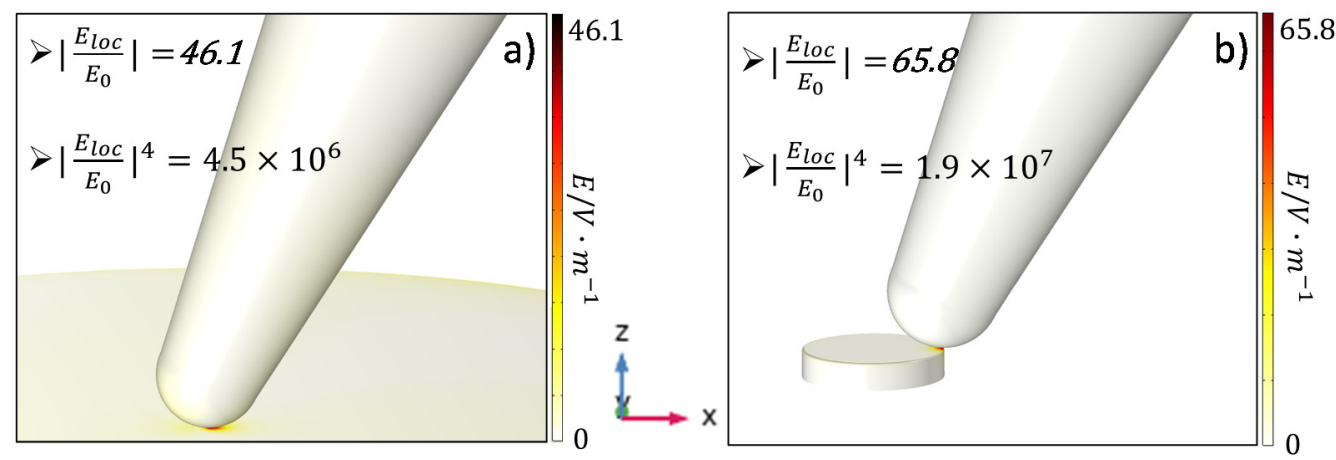

Figure 6. 3D simulation results of (a) Au tip with diameter of $80 \mathrm{~nm}$ on the flat gold substrate; (b) Au tip with diameter of $80 \mathrm{~nm}$ at the edge of a $20 \mathrm{~nm}$ high Au nanodisc with disc diameter of $100 \mathrm{~nm}$. The electric field EF and corresponding TERS EF are presented in the figures respectively.

A comparison on the TERS EF between the 2D and 3D simulations for two specific experimental geometries shown in Figure 6 is demonstrated in Table 1. The tip diameter of $80 \mathrm{~nm}$ and the nanodisc diameter of $100 \mathrm{~nm}$ are set for both 2D and 3D simulation. The excitation wavelength is set as a $p$-polarized $638 \mathrm{~nm}$ with light polarized along the tip long axis. Nevertheless, there are some different settings between the 2D and 3D configurations; for instance, the tip is placed perpendicular to the surface of substrate in 2D geometry, while it is $25^{\circ}$ tilted from the surface normal in $3 \mathrm{D}$. 
Table 1. Comparison on TERS EF between 2D and 3D simulation results for the two specific geometries representing the two experimental configurations.

\begin{tabular}{ccc}
\hline & Tip on Flat Gold & Tip on Edge of Nanodisc \\
\hline 2D simulation & $7.7 \times 10^{6}$ & $8.2 \times 10^{6}$ \\
\hline 3D simulation & $4.5 \times 10^{6}$ & $1.9 \times 10^{7}$ \\
\hline
\end{tabular}

Note that the plasmonic resonance depends on the geometry of the system. Therefore, it is not necessarily true that both $2 \mathrm{D}$ and $3 \mathrm{D}$ plasmonic response will be in resonance at the same excitation wavelength (638 $\mathrm{nm}$ in the present investigation). As can be seen from Table 1, both 2D and 3D simulations shows the same qualitative trends that the TERS EF is higher when the tip is placed on the edge of the nanodisc compared with when the tip is placed on flat gold. However, the relative scale is around 1.1 for $2 \mathrm{D}$ and 4.1 for 3D simulations. Even though we see a perfect quantitative agreement between the 3D simulation results and experiments, the $2 \mathrm{D}$ simulation does offer a qualitative agreement with experiment as well. Considering the huge computational cost of any 3D simulation for large data set, 2D simulation is a very powerful tool providing a fast and systematic understanding for any plasmonic systems.

\section{Summary}

In summary, we investigate the possibility of improving TERS sensitivity via a systematical 2D FEM simulation study for two configurations. The first one consists of a gold conical tip with various diameters placed on a flat gold thin film representing the conventional gap-mode TERS and the other one consists of a gold conical tip with a fixed diameter of $80 \mathrm{~nm}$ placed on a gold nanodisc modified gold thin film. For the second configuration we investigated two possibilities - one with the tip placed at the center of the nanodisc and the second with the tip placed at the edge of the nanodisc. Our simulations clearly demonstrate that the nanodisc substrate provides better TERS sensitivity (both TERS EF and spatial resolution) compared to the conventional gap-mode TERS. Additionally, we also observed that the best TERS performance (strong improvement of both TERS EF and spatial resolution) can be achieved when the tip is placed at the edge of the nanostructures. We also conducted TERS experiments and the corresponding 3D simulation to validate our simulation results and found very good agreement between them. Our model can also be adopted for any other materials. Our results will help to understand and design the high resolution TERS experiments, which is highly pursued in optical nanospectroscopy.

Supplementary Materials: The following are available online at https:/ / www.mdpi.com/2079-499 1/11/2/376/s1, Figure S1: Max. TERS EF and EM loss energy density vs. tip size, Figure S2: Spectral dependence of TERS EF when the tip placed at the edge of the nanodisc, and Figure S3: TERS on monolyer $\mathrm{MoS}_{2}$ on gold nanodisc arrays.

Author Contributions: Conceptualization, M.R.; methodology, M.R., L.H. and T.I.M.; software, L.H.; validation, L.H. and M.R.; formal analysis, L.H.; investigation, L.H. and M.R.; resources, D.R.T.Z.; writing—original draft preparation, L.H. and M.R.; writing—review and editing, T.I.M. and D.R.T.Z.; supervision, M.R. and T.I.M.; project administration, T.I.M. and D.R.T.Z.; funding acquisition, D.R.T.Z. All authors have read and agreed to the published version of the manuscript.

Funding: This research was supported by the SMWK/ESF (RL ESF Hochschule und Forschung 2014-2020 mit Antragsnummer 100380093) and DFG ZA 146/44-1 projects.

Data Availability Statement: The data presented in this study are available on request from the corresponding author. 
Acknowledgments: We would like to thank Marina Lindner for assisting in TERS experiments. We also would like to thank IBM Zurich to prepare the reference gold nanodisc array substrate. We would like to thank A.G Milekhin from Novosibirsk Russia for kindly providing gold nanodisc arrays on Si substrate. The publication costs of this article were funded by the German Research Foundation/DFG and the Technische Universität Chemnitz in the funding programme Open Access Publishing.

Conflicts of Interest: The authors declare no conflict of interest.

\section{References}

1. Chen, C.; Hayazawa, N.; Kawata, S. A 1.7 Nm Resolution Chemical Analysis of Carbon Nanotubes by Tip-Enhanced Raman Imaging in the Ambient. Nat. Commun. 2014, 5, 3312. [CrossRef] [PubMed]

2. Zhong, J.H.; Jin, X.; Meng, L.; Wang, X.; Su, H.S.; Yang, Z.L.; Williams, C.T.; Ren, B. Probing the Electronic and Catalytic Properties of a Bimetallic Surface with 3nm Resolution. Nat. Nanotechnol. 2017, 12, 132-136. [CrossRef] [PubMed]

3. Rahaman, M.; Milekhin, A.G.; Mukherjee, A.; Rodyakina, E.E.; Latyshev, A.V.; Dzhagan, V.M.; Zahn, D.R.T. The Role of a Plasmonic Substrate on the Enhancement and Spatial Resolution of Tip-Enhanced Raman Scattering. Faraday Discuss. 2019, 214, 309-323. [CrossRef] [PubMed]

4. Zhang, R.; Zhang, Y.; Dong, Z.C.; Jiang, S.; Zhang, C.; Chen, L.G.; Zhang, L.; Liao, Y.; Aizpurua, J.; Luo, Y.; et al. Chemical Mapping of a Single Molecule by Plasmon-Enhanced Raman Scattering. Nature 2013, 498, 82-86. [CrossRef] [PubMed]

5. Zhang, C.; Chen, B.Q.; Li, Z.Y. Optical Origin of Subnanometer Resolution in Tip-Enhanced Raman Mapping. J. Phys. Chem. C 2015, 119, 11858-11871. [CrossRef]

6. Chiang, N.; Chen, X.; Goubert, G.; Chulhai, D.V.; Chen, X.; Pozzi, E.A.; Jiang, N.; Hersam, M.C.; Seideman, T.; Jensen, L.; et al. Conformational Contrast of Surface-Mediated Molecular Switches Yields Ångstrom-Scale Spatial Resolution in Ultrahigh Vacuum Tip-Enhanced Raman Spectroscopy. Nano Lett. 2016, 16, 7774-7778. [CrossRef]

7. Kumar, N.; Mignuzzi, S.; Su, W.; Roy, D. Tip-Enhanced Raman Spectroscopy: Principles and Applications. EPJ Tech. Instrum. 2015, 2.

8. Etchegoin, P.G.; Le Ru, E.C. Basic Electromagnetic Theory of Surface Enhanced Raman Spectroscopy; Wiley: Hoboken, NJ, USA, 2010; ISBN 9783527632756

9. Yang, Z.; Aizpurua, J.; Xu, H. Electromagnetic Field Enhancement in TERS Configurations. J. Raman Spectrosc. 2009, 40, 1343-1348. [CrossRef]

10. Ritchie, R.H. Plasma Losses by Fast Electrons in Thin Films. Phys. Rev. 1956, 106, 8. [CrossRef]

11. Steidtner, J.; Pettinger, B. Tip-Enhanced Raman Spectroscopy and Microscopy on Single Dye Molecules with $15 \mathrm{Nm}$ Resolution. Phys. Rev. Lett. 2008, 100, 1-4. [CrossRef] [PubMed]

12. Pettinger, B.; Domke, K.F.; Zhang, D.; Picardi, G.; Schuster, R. Tip-Enhanced Raman Scattering: Influence of the Tip-Surface Geometry on Optical Resonance and Enhancement. Surf. Sci. 2009, 603, 1335-1341. [CrossRef]

13. Stadler, J.; Schmid, T.; Zenobi, R. Nanoscale Chemical Imaging Using Top-Illumination Tip-Enhanced Raman Spectroscopy. Nano Lett. 2010, 10, 4514-4520. [CrossRef] [PubMed]

14. Kumar, N.; Su, W.; Veselý, M.; Weckhuysen, B.M.; Pollard, A.J.; Wain, A.J. Nanoscale Chemical Imaging of Solid-Liquid Interfaces Using Tip-Enhanced Raman Spectroscopy. Nanoscale 2018, 10, 1815-1824. [CrossRef] [PubMed]

15. Richard-Lacroix, M.; Zhang, Y.; Dong, Z.; Deckert, V. Mastering High Resolution Tip-Enhanced Raman Spectroscopy: Towards a Shift of Perception. Chem. Soc. Rev. 2017, 46, 3922-3944. [CrossRef] [PubMed]

16. Rendell, R.W.; Scalapino, D.J. Surface Plasmons Confined by Microstructures on Tunnel Junctions. Phys. Rev. B 1981, $24,3276-3294$. [CrossRef]

17. Zhu, W.; Esteban, R.; Borisov, A.G.; Baumberg, J.J.; Nordlander, P.; Lezec, H.J.; Aizpurua, J.; Crozier, K.B. Quantum Mechanical Effects in Plasmonic Structures with Subnanometre Gaps. Nat. Commun. 2016, 7, 1-14. [CrossRef]

18. Liao, P.F.; Wokaun, A. Lightning Rod Effect in Surface Enhanced Raman Scattering. J. Chem. Phys. 1982, 76, 751-752. [CrossRef]

19. Huang, T.X.; Huang, S.C.; Li, M.H.; Zeng, Z.C.; Wang, X.; Ren, B. Tip-Enhanced Raman Spectroscopy: Tip-Related Issues. Anal. Bioanal. Chem. 2015, 407, 8177-8195. [CrossRef]

20. Ren, B.; Picardi, G.; Pettinger, B. Preparation of Gold Tips Suitable for Tip-Enhanced Raman Spectroscopy and Light Emission by Electrochemical Etching. Rev. Sci. Instrum. 2004, 75, 837-841. [CrossRef]

21. Rodriguez, R.D.; Sheremet, E.; Müller, S.; Gordan, O.D.; Villabona, A.; Schulze, S.; Hietschold, M.; Zahn, D.R.T. Compact Metal Probes: A Solution for Atomic Force Microscopy Based Tip-Enhanced Raman Spectroscopy. Rev. Sci. Instrum. 2012, 83, 123708. [CrossRef]

22. Xu, G.; Liu, Z.; Xu, K.; Zhang, Y.; Zhong, H.; Fan, Y.; Huang, Z. Constant Current Etching of Gold Tips Suitable for Tip-Enhanced Raman Spectroscopy. Rev. Sci. Instrum. 2012, 83, 103708. [CrossRef] [PubMed]

23. Yang, L.K.; Huang, T.X.; Zeng, Z.C.; Li, M.H.; Wang, X.; Yang, F.Z.; Ren, B. Rational Fabrication of a Gold-Coated AFM TERS Tip by Pulsed Electrodeposition. Nanoscale 2015, 7, 18225-18231. [CrossRef] [PubMed]

24. Gao, L.; Zhao, H.; Li, Y.; Li, T.; Chen, D.; Liu, B. Controllable Fabrication of Au-Coated AFM Probes via a Wet-Chemistry Procedure. Nanoscale Res. Lett. 2018, 13, 366. [CrossRef] [PubMed] 
25. Huang, T.X.; Li, C.W.; Yang, L.K.; Zhu, J.F.; Yao, X.; Liu, C.; Lin, K.Q.; Zeng, Z.C.; Wu, S.S.; Wang, X.; et al. Rational Fabrication of Silver-Coated AFM TERS Tips with a High Enhancement and Long Lifetime. Nanoscale 2018, 10, 4398-4405. [CrossRef] [PubMed]

26. Bartolomeo, G.L.; Goubert, G.; Zenobi, R. Tip Recycling for Atomic Force Microscopy-Based Tip-Enhanced Raman Spectroscopy. Appl. Spectrosc. 2020, 74, 1358-1364. [CrossRef]

27. Li, W.; Zhao, X.; Yi, Z.; Glushenkov, A.M.; Kong, L. Plasmonic Substrates for Surface Enhanced Raman Scattering. Anal. Chim. Acta 2017, 984, 19-41. [CrossRef]

28. Chorsi, H.T.; Lee, Y.; Alù, A.; Zhang, J.X.J. Tunable Plasmonic Substrates with Ultrahigh Q-Factor Resonances. Sci. Rep. 2017, 7, 1-9. [CrossRef]

29. Miranda, H.; Rabelo, C.; Cançado, L.G.; Vasconcelos, T.L.; Oliveira, B.S.; Schulz, F.; Lange, H.; Reich, S.; Kusch, P.; Jorio, A. Impact of Substrate on Tip-Enhanced Raman Spectroscopy-A Comparison of Frequency Domain Simulations and Graphene Measurements. arXiv 2020, arXiv:2001.06688.

30. Yuksel, A.; Yu, E.T.; Murthy, J.; Cullinan, M. Effect of Substrate and Nanoparticle Spacing on Plasmonic Enhancement in Three-Dimensional Nanoparticle Structures. J. Micro Nano-Manuf. 2017, 5, 040903. [CrossRef]

31. Tame, M.S.; McEnery, K.R.; Özdemir, Ş.K.; Lee, J.; Maier, S.A.; Kim, M.S. Quantum Plasmonics. Nat. Phys. 2013, 9, 329-340. [CrossRef]

32. Jeon, H.B.; Tsalu, P.V.; Ha, J.W. Shape Effect on the Refractive Index Sensitivity at Localized Surface Plasmon Resonance Inflection Points of Single Gold Nanocubes with Vertices. Sci. Rep. 2019, 9, 13635. [CrossRef] [PubMed]

33. Agrawal, A.; Kriegel, I.; Milliron, D.J. Shape-Dependent Field Enhancement and Plasmon Resonance of Oxide Nanocrystals. J. Phys. Chem. C 2015, 119, 6227-6238. [CrossRef]

34. Benz, F.; Chikkaraddy, R.; Salmon, A.; Ohadi, H.; De Nijs, B.; Mertens, J.; Carnegie, C.; Bowman, R.W.; Baumberg, J.J. SERS of Individual Nanoparticles on a Mirror: Size Does Matter, but so Does Shape. J. Phys. Chem. Lett. 2016, 7, 2264-2269. [CrossRef] [PubMed]

35. Jean-Pierre, B. A Perfectly Matched Layer for the Absorption of Electromagnetic Waves. J. Comput. Phys. 1994, 114, 185-200.

36. McPeak, K.M.; Jayanti, S.V.; Kress, S.J.P.; Meyer, S.; Iotti, S.; Rossinelli, A.; Norris, D.J. Plasmonic Films Can Easily Be Better: Rules and Recipes. ACS Photonics 2015, 2, 326-333. [CrossRef]

37. Milekhin, A.G.; Rahaman, M.; Rodyakina, E.E.; Latyshev, A.V.; Dzhagan, V.M.; Zahn, D.R.T. Giant Gap-Plasmon Tip-Enhanced Raman Scattering of MoS2 Monolayers on Au Nanocluster Arrays. Nanoscale 2018, 10, 2755-2763. [CrossRef]

38. Kolwas, K.; Derkachova, A. Damping Rates of Surface Plasmons for Particles of Size from Nano- to Micrometers; Reduction of the Nonradiative Decay. J. Quant. Spectrosc. Radiat. Transf. 2013, 114, 45-55. [CrossRef]

39. Politano, A.; Formoso, V.; Chiarello, G. Dispersion and Damping of Gold Surface Plasmon. Plasmonics 2008, 3, 165-170. [CrossRef]

40. Zhou, N.; López-Puente, V.; Wang, Q.; Polavarapu, L.; Pastoriza-Santos, I.; Xu, Q.H. Plasmon-Enhanced Light Harvesting: Applications in Enhanced Photocatalysis, Photodynamic Therapy and Photovoltaics. RSC Adv. 2015, 5, 29076-29097. [CrossRef]

41. Fang, P.-P.; Li, J.-F.; Yang, Z.-L.; Li, L.-M.; Ren, B.; Tian, Z.-Q. Optimization of SERS Activities of Gold Nanoparticles and Gold-core-Palladium-shell Nanoparticles by Controlling Size and Shell Thickness. J. Raman Spectrosc. 2008, 39, $1679-1687$. [CrossRef]

42. Tiggesbäumker, J.; Köller, L. Blue Shift Ofthe Mie Plasma Frequency in Ag Clusters and Particles. Phys. Rev. A 1993, 48, 1749-1752.

43. Zhu, S.; Chen, T.P.; Cen, Z.H.; Goh, E.S.M.; Yu, S.F.; Liu, Y.C.; Liu, Y. Split of Surface Plasmon Resonance of Gold Nanoparticles on Silicon Substrate: A Study of Dielectric Functions. Opt. Express 2010, 18, 21926. [CrossRef] [PubMed]

44. Downes, A.; Salter, D.; Elfick, A. Heating Effects in Tip-Enhanced Optical Microscopy. Opt. Express 2006, 14, 5216. [CrossRef] [PubMed]

45. Downes, A.; Salter, D.; Elfick, A. Finite Element Simulations of Tip-Enhanced Raman and Fluorescence Spectroscopy. J. Phys. Chem. B 2006, 110, 6692-6698. [CrossRef] [PubMed]

46. Alvarez, M.M.; Khoury, J.T.; Schaaff, T.G.; Shafigullin, M.N.; Vezmar, I.; Whetten, R.L. Optical Absorption Spectra of Nanocrystal Gold Molecules. J. Phys. Chem. B 1997, 101, 3706-3712. [CrossRef]

47. Andrew Downes; Donald Salter; Alistair Ilfick Simulations of Tip-Enhanced Optical Microscopy Reveal Atomic Resolution. J. Microsc. 2008, 229, 184-188. [CrossRef]

48. Shi, X.; Coca-López, N.; Janik, J.; Hartschuh, A. Advances in Tip-Enhanced near-Field Raman Microscopy Using Nanoantennas. Chem. Rev. 2017, 117, 4945-4960. [CrossRef]

49. Kelly, K.L.; Coronado, E.; Zhao, L.L.; Schatz, G.C. The Optical Properties of Metal Nanoparticles: The Influence of Size, Shape, and Dielectric Environment. J. Phys. Chem. B 2003, 107, 668-677. [CrossRef]

50. Campos, A.; Troc, N.; Cottancin, E.; Pellarin, M.; Weissker, H.C.; Lermé, J.; Kociak, M.; Hillenkamp, M. Plasmonic Quantum Size Effects in Silver Nanoparticles Are Dominated by Interfaces and Local Environments. Nat. Phys. 2019, 15, 275-280. [CrossRef]

51. Christensen, T.; Yan, W.; Jauho, A.P.; Soljačić, M.; Mortensen, N.A. Quantum Corrections in Nanoplasmonics: Shape, Scale, and Material. Phys. Rev. Lett. 2017, 118, 1-6. [CrossRef]

52. Barbry, M.; Koval, P.; Marchesin, F.; Esteban, R.; Borisov, A.G.; Aizpurua, J.; Sánchez-Portal, D. Atomistic Near-Field Nanoplasmonics: Reaching Atomic-Scale Resolution in Nanooptics. Nano Lett. 2015, 15, 3410-3419. [CrossRef] [PubMed]

53. Milekhin, A.G.; Kuznetsov, S.A.; Sveshnikova, L.L.; Duda, T.A.; Milekhin, I.A.; Rodyakina, E.E.; Latyshev, A.V.; Dzhagan, V.M.; Zahn, D.R.T. Surface-Enhanced Infrared Absorption by Optical Phonons in Nanocrystal Monolayers on Au Nanoantenna Arrays. J. Phys. Chem. C 2017, 121, 5779-5786. [CrossRef] 
54. Rahaman, M.; Rodriguez, R.D.; Plechinger, G.; Moras, S.; Schüller, C.; Korn, T.; Zahn, D.R.T. Highly Localized Strain in a MoS2/Au Heterostructure Revealed by Tip-Enhanced Raman Spectroscopy. Nano Lett. 2017, 17, 6027-6033. [CrossRef] [PubMed]

55. Milekhin, I.A.; Rahaman, M.; Anikin, K.V.; Rodyakina, E.E.; Duda, T.A.; Saidzhonov, B.M.; Vasiliev, R.B.; Dzhagan, V.M.; Milekhin, A.G.; Latyshev, A.V.; et al. Resonant Tip-Enhanced Raman Scattering by CdSe Nanocrystals on Plasmonic Substrates. Nanoscale Adv. 2020, 2, 5441-5449. [CrossRef] 